\title{
A note on precision-preserving compression of scientific data
}

\author{
Rostislav Kouznetsov ${ }^{1,2}$ \\ ${ }^{1}$ Finnish Meteorological Institute, Helsinki, Finland \\ ${ }^{2}$ Obukhov Institute for Atmospheric Physics, Moscow, Russia \\ Correspondence: Rostislav Kouznetsov (rostislav.kouznetsov@fmi.fi) \\ Received: 14 July 2020 - Discussion started: 30 July 2020 \\ Revised: 17 November 2020 - Accepted: 4 December 2020 - Published: 22 January 2021
}

\begin{abstract}
Lossy compression of scientific data arrays is a powerful tool to save network bandwidth and storage space. Properly applied lossy compression can reduce the size of a dataset by orders of magnitude while keeping all essential information, whereas a wrong choice of lossy compression parameters leads to the loss of valuable data. An important class of lossy compression methods is so-called precisionpreserving compression, which guarantees that a certain precision of each number will be kept. The paper considers statistical properties of several precision-preserving compression methods implemented in NetCDF Operators (NCO), a popular tool for handling and transformation of numerical data in NetCDF format. We compare artifacts resulting from the use of precision-preserving compression of floating-point data arrays. In particular, we show that a popular Bit Grooming algorithm (default in NCO until recently) has suboptimal accuracy and produces substantial artifacts in multipoint statistics. We suggest a simple implementation of two algorithms that are free from these artifacts and have double the precision. One of them can be used to rectify the data already processed with Bit Grooming.

We compare precision trimming for relative and absolute precision to a popular linear packing (LP) method and find out that LP has no advantage over precision trimming at a given maximum absolute error. We give examples when LP leads to an unconstrained error in the integral characteristic of a field or leads to unphysical values.

We analyze compression efficiency as a function of target precision for two synthetic datasets and discuss precision needed in several atmospheric fields.

Mantissa rounding has been contributed to NCO mainstream as a replacement for Bit Grooming. The Appendix contains code samples implementing precision trimming in Python3 and Fortran 95.
\end{abstract}

\section{Introduction}

Resolutions and the level of details of processes simulated with geoscientific models increase together with the increase in computing power available. A corresponding increase in the size of datasets needed to drive the models and the datasets produced by the models makes the problem of transferring and archiving the data more and more acute.

The data are usually produced and stored as floating-point numbers implemented in most of computer systems according to the IEEE 754-1985 standard (ANSI/IEEE, 1985). The standard offers two formats: single precision and double precision. The data in these formats have precisions of approximately 7 and 16 significant decimal figures and occupy 32 and 64 bits per value in a memory or on a disk.

The problem of storing and transferring the data is normally addressed in three directions: increase in storage capacities and network bandwidth, reduction in the number of stored or transferred values by careful selection of required variables and a wise choice of the archiving policies, and the application of various data compression techniques. We will focus on the latter.

Lossless compression algorithms map a data array to a smaller size in a way that the original data can be restored exactly. The algorithms are efficient for datasets of low information entropy rate, e.g., those that have a lot of repeating patterns.

Many scientific datasets have only a few (or even a few tens of) percent accuracy and thus require much less than seven decimals to represent a value. When represented with standard formats, such datasets have seemingly random numbers at less significant places, i.e., have high information entropy. As a result, the application of lossless compression algorithms to such datasets does not lead to a substantial reduc- 
tion in the dataset size. Transformations of a data array reducing its information entropy while introducing acceptable distortions pose the basis for lossy compression algorithms. A transformation can make the array smaller or can facilitate the performance of subsequent lossless compression. Note that the meaning of "acceptable distortion" depends on the data and/or the intended application.

An often used method of lossy compression is linear packing, when the original floating-point data are mapped to a shorter-length integer data by a linear transformation. The method, introduced in GRIB (GRidded Binary) format approved back in 1985 by the World Meteorological Organization and intended for use for gridded weather data (Stackpole, 1994), is still in use in subsequent standards. A similar technique has been applied for NetCDF format according to the CF (Climate and Forecast) conventions (http:// cfconventions.org, last access: 18 January 2021). The linear packing into a shorter data type allows for reduction in the storage space even without involving subsequent data compression. The method has been working well for the data of a limited dynamic range (within 1-3 orders of magnitude); however it causes poor representation for the data that have a larger dynamic range or too skewed a distribution of values.

Another class of lossy compression methods, so-called precision-preserving compression, is designed to guarantee a certain relative precision of the data. Setting a certain number of least significant bits of the floating-point numbers in a data array to a prescribed value (trimming the precision) substantially reduces the entropy of the data making lossless compression algorithms much more efficient. A combination of precision trimming and a lossless compression constitutes an efficient method of lossy compression with wellcontrolled loss of relative precision. Unlike packing, precision trimming maps an array of floating-point numbers to an array of floating-point numbers that do not need any special treatment before use.

Zender (2016) implemented precision trimming in a versatile data-processing tool set called NetCDF Operators (NCO; http://nco.sourceforge.net, last access: 7 December 2020), enabling the internal data compression features of the NetCDF4 format (https://www.unidata.ucar.edu/ software/netcdf, last access: 7 December 2020) to work efficiently. The tool set allows a user to chose the required number of decimals for each variable in a file, so precision trimming can be applied flexibly depending on the nature of the data. It was quickly noticed that a simple implementation of precision trimming by setting non-significant bits to 0 (bit shaving) introduces undesirable bias to the data. As a way to cope with that bias Zender (2016) introduced a Bit Grooming algorithm that alternately shaves (to 0 ) and sets (to 1 ) the least significant bits of consecutive values. A detailed review of the compression methods used for scientific data as well as the analysis of the influence of the compression parameters on compression performance can be found in the paper by Zender (2016) and references therein.
While comparing spatial structure functions of stratospheric ozone mixing ratio from satellite retrievals to those obtained from the Silam chemistry-transport model (http: //silam.fmi.fi, last access: 18 January 2021) and compressed with NCO, we spotted a substantial distortion introduced by Bit Grooming into the structure function of the modeled fields. In the current note we investigate the cause of this distortion. We consider several precision-trimming algorithms and the inaccuracies they introduce. We suggest an implementation of mantissa rounding that provides double the accuracy of the data representation as Bit Grooming with the same number of data bits and does not introduce major artifacts in two-point statistics. In addition, we suggest a method of recovering the bit-groomed data.

The paper is organized as follows. The next section formulates the concept of precision trimming and describes various methods for it and their implementation. Section 3 analyzes the effect of precision-trimming methods on the introduced errors. Section 4 illustrates the effect of precision trimming on the statistics of two synthetic datasets. Besides relative-precision trimming we consider the properties of the absolute-precision trimming (Sect. 5) and linear packing (Sect. 6). The effect of trimming and linear packing on the storage size reduction is analyzed in Sect. 7. Parameters of precision trimming suitable for several variables from atmospheric modeling are discussed in Sect. 8. Conclusions are summarized at the end of the paper. Appendix A contains example implementations of subroutines for precision trimming.

\section{Precision-trimming methods}

Hereafter we will consider a single-precision floating-point representation, although all the conclusions can be extrapolated to arbitrary-precision numbers. A single-precision number according to ANSI/IEEE (1985) consists of 32 bits: 1 bit for the sign, 8 bits for the exponent, and 23 bits for the mantissa (also called the "fraction"):

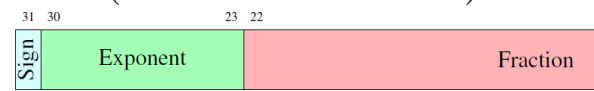

The implicit most significant bit (MSB) of the mantissa is 1 ; therefore the mantissa has 24-bit resolution. $M$ bits of the mantissa allow for distinguishing between any two numbers $a$ and $b$ if

$2 \frac{|a-b|}{|a|+|b|}>2^{-M}$

therefore the representation provides $2^{-24} \approx 6 \times 10^{-8}$ relative precision or about seven decimals.

If the data have smaller precision, the least significant bits (LSBs) of the mantissa contain seemingly random information, which makes lossless-compression algorithms inefficient. If one trims the precision, i.e., replaces those bits with a pre-defined value, the overall entropy of the dataset reduces 
and the efficiency of compression improves. Therefore the problem of trimming LSBs can be formulated as follows: given an array of floating-point numbers, transform it so that the resulting values have all but $N$ LSBs of their mantissa set to a prescribed combination, while the difference from the original array is minimal.

Hereafter we will use the term "keep bits" for the $N$ most significant bits of the mantissa, which we use to store the information from the original values, and "tail bits" for the remaining bits of a mantissa that we are going to set to a prescribed value. The resulting precision is characterized by the number of bits of the mantissa that we are going to keep.

The following methods have been considered:

- shave - set all tail bits to 0

- set-set all tail bits to 1

- groom - set all tail bits to 1 or to 0 in alternate order for every next element of the array

- halfshave - set all tail bits to 0 except for the most significant one of them, which is set to 1

- round - round the mantissa to the nearest value that has zero tail bits. Note that this rounding affects keep bits and might affect the exponent as well.

The first three of these were implemented in NCO some years ago and are described well by Zender (2016). They can be implemented by applying of bit masks to the original floating-point numbers. Halfshave and round have been recently introduced into NCO. Halfshave is trivial to implement by applying an integer bit mask to a floating-point number: the first one applies the shave bit mask and then sets the MSB of tail bits to 1 .

Rounding can be implemented with integer operations (Milan Klöwer, personal communication, July 2020). Consider an unsigned integer that has all bits at 0 , except for the most significant one of the tail bits. Adding this integer to the original value $u$, treated as an unsigned integer, would not change the keep bits if $u$ has 0 in the corresponding bit, and bit shaving of the sum will produce the same result as bit shaving the original value, i.e., $u$ rounded towards 0 . If $u$ has 1 in the corresponding bit, the carry bit will propagate to the fraction or even to the exponent. Bit shaving the resulting value is equivalent to rounding $u$ towards infinity. In both cases the result is half-to-infinity rounding, i.e., rounding to the nearest discretization level, and the value that is exactly in the middle of a discretization interval would be rounded away from 0 .

Rounding half to infinity introduces a small bias away from 0 on average. The magnitude of the bias is half of the value of the least significant bit of the original number, i.e., about $10^{-8}$ for the single precision. For many applications such biases can be safely neglected. However, if one applies such a procedure in a sequence trimming the tail bits one by one, the result will be equivalent to rounding to infinity; i.e., it will have an average bias of the value of the most significant of the tail bits. A rigorous procedure of rounding half to even (a default rounding mode in IEEE 754) is free from biases and should be used.

The implementations of the abovementioned bit trimming and rounding procedures are given in Appendix A.

\section{Quantification of errors}

To characterize the trimming we will use the number of explicit bits kept in the mantissa of a floating-point number. Therefore "bits $=1$ " means that the mantissa keeps 1 implicit and 1 explicit bits for the value and the remaining 22 bits have been reset to a prescribed combination.

When rounding to a fixed quantum (e.g., to integer), the magnitude of the maximal introduced error is a function of the quantum. The error is one quantum for one-sided rounding (up, down, towards 0 , away from 0 ) and half of the quantum for rounding to the nearest value. With precisionpreserving compression, the quantum depends on the value itself. For a value $v$ discretized with $M$ bits of the mantissa kept $(M-1$ keep bits) the quantum is the value of the least significant bit of keep bits, i.e., at least $\left|2^{1-M} v\right|$, and at most $\left|2^{-M} v\right|$ depending on how far $v$ is from the maximum power of 2 that is less than $v$. Note that the quantization levels for the halfshave method are at midpoints of the intervals between the levels for other methods.

The shave method implements rounding towards 0 . The set method is almost rounding away from 0 . They introduce an error of at most a full quantum to each value with a mean absolute error of a half-quantum. The groom method being alternately shave and set has the same absolute error as those but is almost unbiased on average. The round method takes into account the value of the MSB of tail bits and therefore introduces an error of at most a half-quantum with a mean absolute error of a quarter of a quantum. The same applies to the halfshave method, since its levels are midpoints of the range controlled by the tail bits of the original value. Note that for the mean absolute error estimate we assume that the tail bits have an equal chance of being 0 or 1 .

The margins of error introduced by the methods for different numbers of mantissa bits are summarized in Table 1. As one can see, the round and halfshave methods allow for half the error for the same number of keep bits or one less keep bit to guarantee the same accuracy as shave, set, or groom.

The results of applying precision trimming with various numbers of keep bits are illustrated in Fig. 1, where the 1:1 line is marked as "ref". The set method is not shown, to reduce the number of lines in the panels. For the shown range and keep bits of $8,4,6$, and 2 , the discrete levels are separated by $0.5,2,8$, and 32 , respectively. The levels of halfshave have a half-discrete offset with respect to the others. For eight keep bits (Fig. 1a) on the scale of the plots the distortion is almost 
(a) 8 keep bits
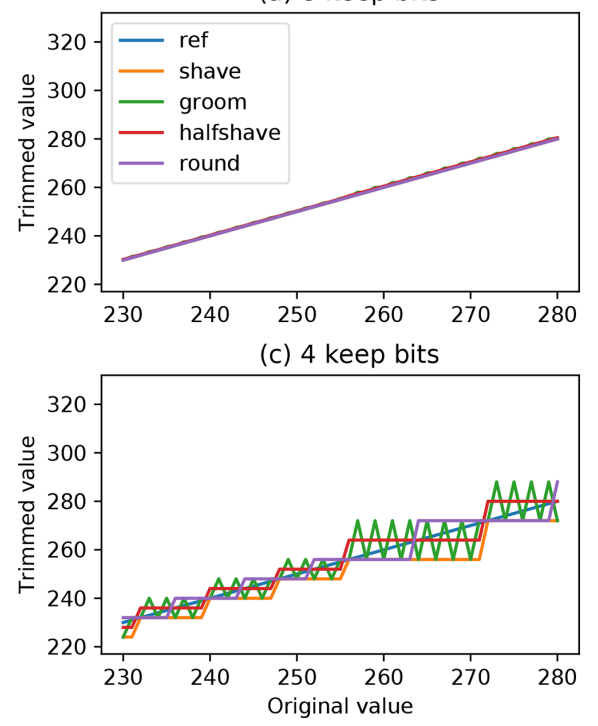

(b) 6 keep bits

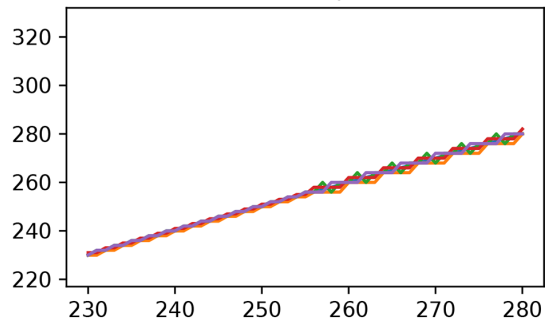

(d) 2 keep bits

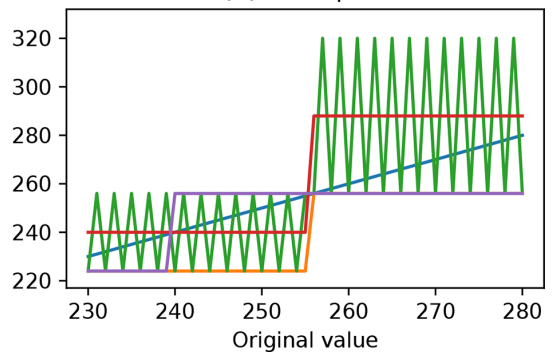

Figure 1. Trimmed values as a function of original values when trimmed to different numbers of keep bits. The lines correspond to the different trimming methods.

Table 1. The discretization level increment for different numbers of the mantissa bits kept and the maximum relative error introduced by trimming the precision with various methods.

\begin{tabular}{|c|c|c|c|c|c|c|}
\hline \multirow[t]{2}{*}{ Mantissa size (bit) } & \multirow[t]{2}{*}{ Keep bits } & \multicolumn{3}{|c|}{$\begin{array}{l}\text { Discretization level increment } \\
\text { as a fraction of the value }\end{array}$} & \multicolumn{2}{|c|}{ Max relative error } \\
\hline & & & & & Shave, Set, Groom & Round, Halfshave \\
\hline 1 & 0 & 0.5 & $\ldots$ & 1 & $100 \%$ & $50 \%$ \\
\hline 2 & 1 & 0.25 & $\ldots$ & 0.5 & $50 \%$ & $25 \%$ \\
\hline 3 & 2 & 0.125 & $\ldots$ & 0.25 & $25 \%$ & $12.5 \%$ \\
\hline 4 & 3 & 0.0625 & $\ldots$ & 0.125 & $12.5 \%$ & $6.25 \%$ \\
\hline 5 & 4 & 0.03125 & $\ldots$ & 0.0625 & $6.25 \%$ & $3.1 \%$ \\
\hline 6 & 5 & 0.015625 & $\ldots$ & 0.03125 & $3.1 \%$ & $1.6 \%$ \\
\hline 7 & 6 & 0.0078125 & $\ldots$ & 0.015625 & $1.6 \%$ & $0.8 \%$ \\
\hline 8 & 7 & 0.00390625 & $\ldots$ & 0.0078125 & $0.8 \%$ & $0.5 \%$ \\
\hline 9 & 8 & 0.001953125 & $\ldots$ & 0.00390625 & $0.5 \%$ & $0.25 \%$ \\
\hline
\end{tabular}

invisible, whereas for two keep bits the difference is easily seen. Note the increase in the quantum size at 256 due to the increment of the exponent.

It was pointed by Zender (2016) the shave method introduces a bias towards 0 , which might be unwanted in some applications (set introduces the opposite bias). The idea of Bit Grooming (groom) is to combine these two biased trimming procedures to get on average unbiased fields.

However, it has been overlooked that Bit Grooming introduces an oscillating component that affects multipoint statistics. With Bit Grooming, the quantization of a value in an array depends not only on the value itself but also on its index in the array. As a result of this, the absolute difference between two values with even and odd indices will become positively biased on average, while the absolute difference between two points with both even (or both odd) indices will stay unbiased.

One can note that the result of applying the halfshave procedure with the same number of keep bits to a bit-groomed field is equivalent to applying it to the original field. Therefore halfshave can be regarded as a method to halve the error and remove the artifacts of Bit Grooming.

\section{Examples}

Consider an array of $N$ floating-point numbers $u_{i}$ and its precision-trimmed version $v_{i}$. To illustrate the performance of the algorithms we will consider the normalized root-mean- 
(a) Correlated signal

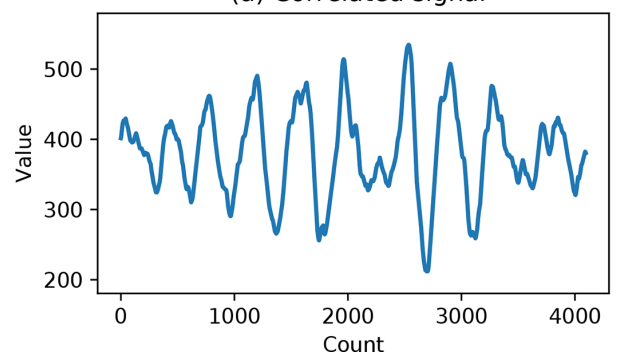

(b) Uncorrelated signal

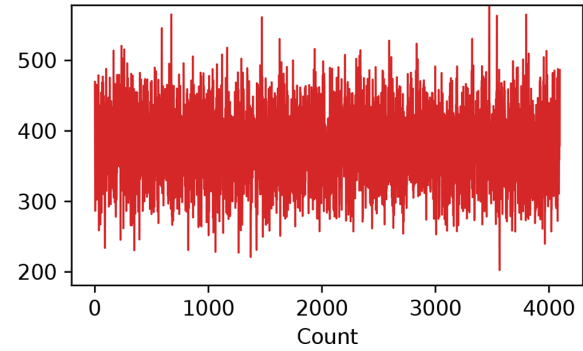

Figure 2. The signals used for illustration (a) correlated and (b) uncorrelated.

(a) shave

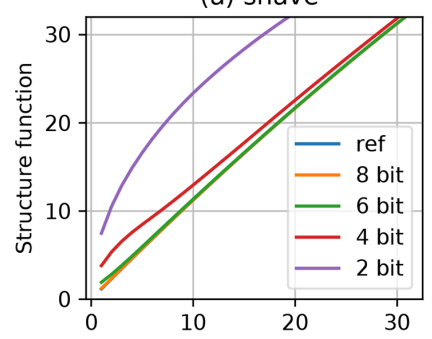

(d) round

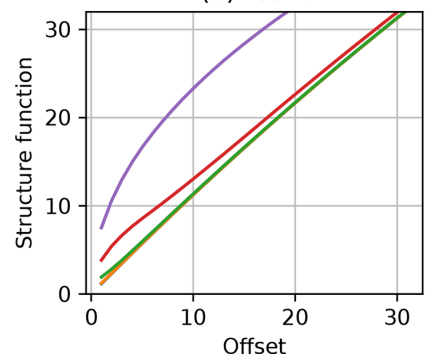

(b) groom

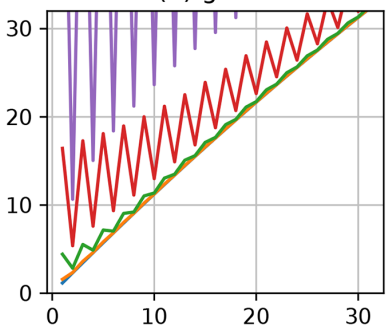

(e) groomhalf

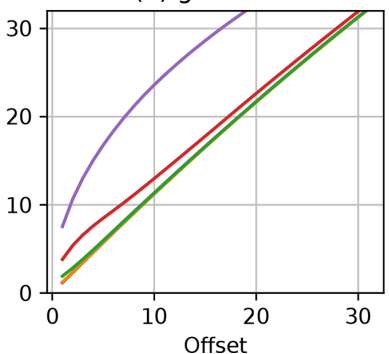

(c) halfshave

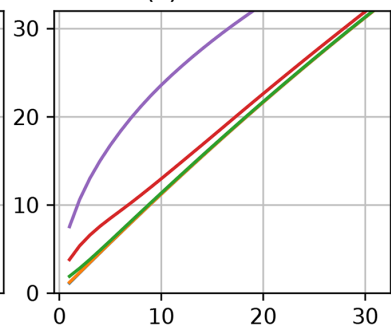

(f) groomav

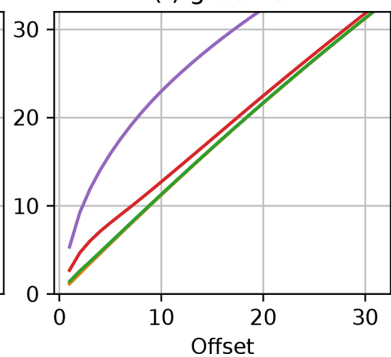

Figure 3. The structure functions (Eq. 3) of the correlated signal shown in Fig. 2a, processed with different precision trimmings.

Table 2. NRMSE (normalized root-mean-square error) of the signal in Fig. 2 a after trimming precision.

\begin{tabular}{lrrr}
\hline & Two keep bits & Four keep bits & Six keep bits \\
\hline Shave & 0.09800 & 0.02479 & 0.00619 \\
Groom & 0.09941 & 0.02478 & 0.00620 \\
Halfshave & 0.04968 & 0.01231 & 0.00310 \\
Round & 0.04927 & 0.01252 & 0.00311 \\
Groomhalf & 0.04968 & 0.01231 & 0.00310 \\
Groomav & 0.04870 & 0.01141 & 0.00280 \\
\hline
\end{tabular}

square error (NRMSE) introduced by precision trimming,

$\mathrm{NRMSE}=\sqrt{\frac{1}{N} \sum_{i=1}^{N} \frac{\left(u_{i}-v_{i}\right)^{2}}{u_{i}^{2}}}$, and a distortion to the structure function of the precisiontrimmed fields, which is defined as follows:

$X(r)=\sqrt{\frac{1}{N-r} \sum_{i=r}^{N-r}\left(u_{i}-v_{i+r}\right)^{2}}$,

where the integer argument $r$ is called offset and can be a spatial or temporal coordinate.

To illustrate the features of precision trimming, we will use two synthetic arrays: a random process with a " -2 "- -power spectrum (Fig. 2a) and random Gaussian noise (Fig. 2b). The former is a surrogate of a geophysical signal with high autocorrelation, whereas the latter corresponds to a signal with a large stochastic component. Both signals have identical variance, which is controlled cut-off at low-frequency components of the correlated signal and by the variance parameter of the uncorrelated signal. To simplify the analysis we have added a background that is 8 times the signal's standard deviation. Each array is 32768 values long. The exact code used to generate the signals and all the figures of this paper can be found in the Supplement. 
(a) shave

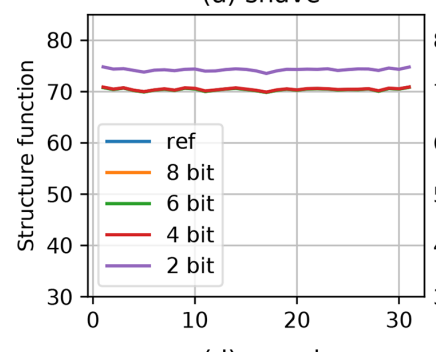

(d) round

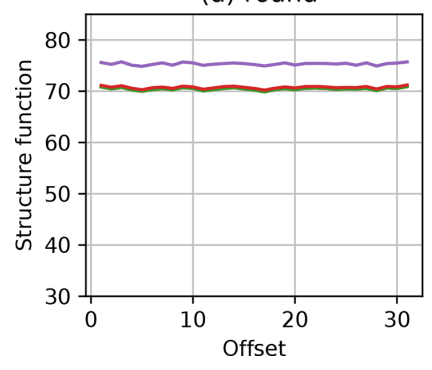

(b) groom

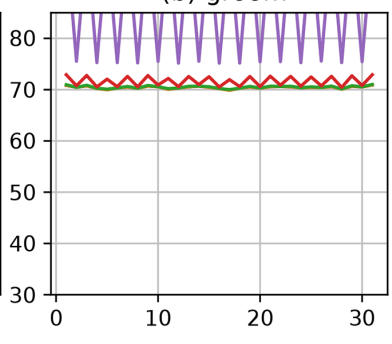

(e) groomhalf

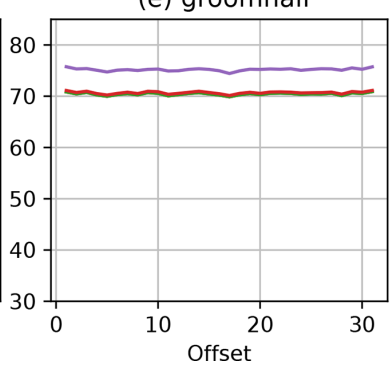

(c) halfshave

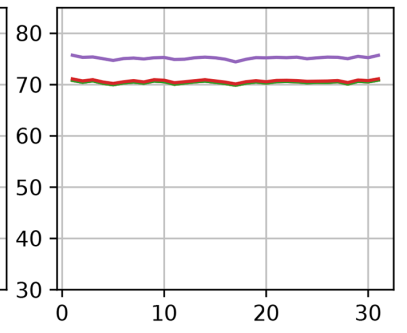

(f) groomav

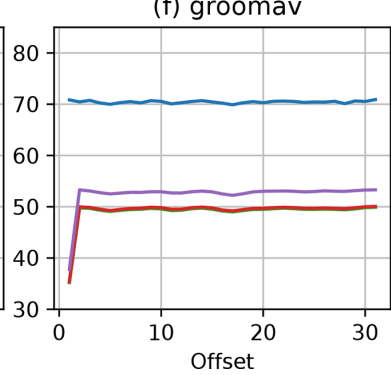

Figure 4. The structure function (Eq. 3) of the signal shown in Fig. 2b, processed with different precision trimmings.

The NRMSE of the signal in Fig. 2a after precision trimming with the considered methods is summarized in Table 2 . The results fully agree with Table 1 : the NRMSE introduced by every method is about half of the maximum error for a single value. Along with abovementioned methods we have added two ways of rectifying bit-groomed values: groomhalf, where we apply halfshave to the bit-groomed array, and groomav, where a simple two-point moving average is applied to the data.

As expected, groomhalf results in exactly the same values as halfshave and therefore has identical NRMSE. The scores for halfshave and round are slightly different due to the limited sample size and limited number of discrete levels for the samples. It is notable that for smooth signals like the one in Fig. 2a, the moving average of bit-groomed values gives a smaller NRMSE than for all other methods. The reason is in the smoothness of the signal, which has well-correlated adjacent points, while bit-trimming errors are less correlated.

For the signal with a " -2 "-power spectrum (Fig. 2a) the structure function is linear. The structure functions of the signal processed with trim-precision are given in Fig. 3. All panels have the structure function of the original signal (ref curve) plotted along with curves for the processed signal. Since the structure function is not sensitive to the offsets, the plots for shave, halfshave, and groomhalf (panels a, c, and e) are identical. Panel d differs slightly form them due to statistical differences mentioned above. The Bit Grooming algorithm produces quite large peaks at odd offsets, whereas the values at even offsets are equal to the corresponding values for shave or halfshave.

As a way to compensate for the artifacts of Bit Grooming without introducing additional bias one could apply moving average (Charles Zender, personal communication
July 2020). For a smooth signal, the running average produces a structure function that is closer to ref than one for the mantissa rounding. The steeper structure function for smaller offsets in Fig $3 \mathrm{f}$ is a result of smoothing by the moving average.

The situation is different for the uncorrelated random signal from Fig. 2b, whose structure function is given in Fig. 4. For this signal the reference structure function is flat and the remaining oscillations are due to the sampling errors. As in the previous case, the increase in the number of keep bits makes the structure function closer to the reference one, but the offset also depends strongly on the location of the background and the variance with respect to the discretization levels. As in the case of the smooth signal, Bit Grooming produces oscillating distortions of the structure function, which can be removed by applying halfshave.

The moving average notably reduces the variance of the uncorrelated signal, and the structure function becomes lower. A dip at the unity offset at Fig $3 \mathrm{f}$ is caused by a correlation between neighboring points introduced by the moving average. Therefore the moving average for poorly correlated signals is more damaging than Bit Grooming.

\section{Keeping absolute precision}

Some applications require a given absolute precision, i.e the acceptable error of data representation is expressed in absolute units rather than as a fraction of a value. For such data, when applying a rounding precision-trimming procedure, one can specify the value of the least significant keep bit, rather than the fixed number of mantissa bits, and therefore ensure that the absolute error of the representation will 
be within a half of that value. The latter method is called the "decimal significant digit" (DSD) method in NCO (Zender, 2016). Since both relative- and absolute-precision-trimming methods reset least significant bits of floating-point values, the statistical properties of the distortions introduced by these methods are similar.

If the required accuracy of a variable is given in terms of both relative and absolute precision, precision trimming with a limit for both the least significant keep-bit number and the least significant keep-bit value can be used. This can be achieved by sequential application of the two trimming procedures.

\section{Precision of linear packing}

Linear packing (LP) is a procedure of applying a linear transformation that maps the range of the original values onto the representable range of an integer data type and rounds and stores the resulting integers. The parameters of the transformation - the offset and scale factor - have to be stored for subsequent recovery of the data. The method itself leads to a reduction in the dataset size if the integer size is shorter than the original floating-point size. Applying a lossless compression to the resulting integer array usually leads to further reduction in size.

When applying LP to a dataset one has to select the parameters of the transformation and a size of the integer data type (bitsperValue parameter). This can be done in two ways:

1. One can specify bitsPerValue and map the range of an array onto the representable range. In this case a full array has to be analyzed prior to the packing, and the absolute precision of the representation will be controlled by the difference between the maximum and minimum values of the array. This approach is acceptable when the encoded parameter is known to have a certain range of values; then the resulting errors can be reliably constrained.

2 . One can explicitly specify the required absolute precision of the representation by pre-defining the scale factor. Then a number of bitsPerValue has to be taken with a sufficient margin to ensure that all values can be mapped onto the representable range.

In both cases LP controls the absolute error of the representation, which makes LP suboptimal for the data that require a constraint for a relative error. If a dataset has a large dynamic range (many orders of magnitude), the number of bitsPerValue might become larger than the bit size of the original floating-point values, if they are at all representable with a specific output format (e.g., 32 bits for NetCDF).

GRIB format allows for applying LP to individual gridded 2D fields, and the bitsPerValue parameter can be se- lected to any value in the range of 0-255 (often only 0-63 are implemented). Therefore LP parameters can be optimized for each field to provide the required absolute precision. Writing of large GRIB datasets can be done field by field in a single pass. The approach has been widely used and works well for meteorological data. Recent GRIB2 standards enable a lossless data compression of packed data with the CCSDS/AEC (Consultative Committee for Space Data Systems/Adaptive Entropy Coding) algorithm, which provides further reduction in the packed data size for a typical output of a numericalweather-prediction model by about a half $(50 \%-60 \%$ of reduction in our experience). This compression alleviates the use of redundant bitsPerValue when the absolute precision is specified (option 2 above), and therefore the GRIB2 implementation of LP is good for data of a limited dynamic range and a requirement for absolute precision.

LP for the classic NetCDF format has additional implications. The format provides a choice of 8,16 , or 32 bits per value for integers, and the integers are signed. According to the CF conventions (http://cfconventions.org; last access: 19 January 2021), LP applies the same linear transformation parameters for the whole variable in a file which may contain many levels and/or time steps. Many implementations (e.g., the ncpdq tool from NCO) use option 1 from above, therefore requiring two passes through the dataset, which might require a consideration of memory constraints. This way usually does not lead to noticeable artifacts for common meteorological variables. However, if the original field has a large dynamic range or has a substantially skewed distribution, the errors in LP can be huge.

Consider a gridded field of in-air concentration of a pollutant continuously emitted from a point source in the atmosphere. The maximum value of such a field would depend on a grid resolution, and the number of bits needed to pack such a field with a given relative or absolute accuracy has to be decided on a case-by-case basis. Moreover, if one needed to integrate such a field to get the total emitted mass of the pollutant, the integral of the unpacked field could be very different from the integral of the original field. If the zero value of the original field can be exactly represented as a packed value, a substantial fraction of mass can be hidden in nearzero values. If the zero value of the original field is mapped to a ("small") non-zero value, the total mass, obtained from integration of the unpacked field, would depend on the domain size.

Rounding errors in linear packing can lead to unphysical values. Consider a single-precision field (of, e.g., humidity) that has values between 0 and 0.99999535 . If one packs it to an 8-bit signed integer (byte) with the standard procedure of $\mathrm{NCO}$ (as in version 4.9.4) and unpacks it back with the same $\mathrm{NCO}$, one obtains $-2.980232 \times 10^{-8}$ as the minimum value instead of 0 . The negative value results from rounding errors and a finite precision of the packing parameters. An unphysical value used in a model might lead to arbitrary results. If the variable has a specified valid_range attribute, $\mathrm{CF}$ 
conventions prescribe treating the corresponding values as missing.

Therefore we conclude that LP produces acceptable results when the required margin for absolute errors is within a few orders of the field's maximum value and if the valid range of a variable covers the actual range with that margin. In a general case, the application of LP might lead to errors of arbitrary magnitude or can make valid data invalid.

A useful application of the packing parameters is scaling or unit conversion for the data that already have trimmed precision. If one multiplies precision-trimmed data with a factor that differs form a power of 2, the scaling also affects the tail bits and the compression efficiency reduces. However, if one scales the data by altering the scale_factor attribute of a NetCDF file, the stored array stays intact and well compressible, and CF-aware readers will apply the scaling on reading.

\section{Compressing precision-trimmed data}

To give an example of the storage-space savings due to compression we compressed the test arrays shown in Fig. 2 processed with mantissa rounding and plotted the compresseddata size as a function of the number of keep bits. The size is normalized with the storage size of the original array ( $32768 \times 4$ bytes). We used three compression methods available from the standard Python library: zlib (the same as used with NetCDF4 compression) with level 6, bzip2, and lzma. The latter two are more expensive computationally but provide a higher compression ratio and can be applied to compress the raw binary data files or used in future data formats.

To emulate the compression used with NetCDF4 we have applied shuffling to the bit-trimmed arrays. Shuffling is a procedure of re-arrangement of the data bytes in a floating-point array, so they get ordered sequentially: all first bytes of each number, then all second bytes, etc. The procedure is known to improve the compression ratio in most cases. Shuffling has been available in the NetCDF4 library and is applied in NCO in writing compressed variables.

Figure 5 shows the compression ratio for the precisiontrimmed test signals with (dashed lines) and without (solid lines) shuffling processed with three compression methods. We used mantissa rounding with the number of keep bits from 0 to 23 , i.e., the full range of possible trimmings for single-precision floats. To better visualize the full range of compression ratios we plot them in both linear and logarithmic scales. For a reference the compressed-size of the same arrays packed with linear packing to 8- and 16-bit signed integers is shown. The packed data are attributed to the number of keep bits corresponding to the same maximum absolute error.

For all algorithms the reduction in keep bits leads to the reduction in compressed size. The reduction is nearly linear for the uncorrelated signal and faster than linear for the correlated signal, since more significant bits of the latter are less likely to differ between neighboring points. For the example arrays the monotonicity breaks between 0 and 1 keep bits since for 0 keep bits, the rounding procedure alters the exponent, which stays constant otherwise. Some of the curves have a noticeable dip in the compressed size at 7 and 15 keep bits, corresponding to byte boundaries. The dip is mostly pronounced for zlib compression.

Shuffling substantially improves the compression efficiency and leads to a twice smaller resulting size for our datasets for all compression methods, including linear packing to 16 bits. The reason is that general-purpose compression algorithms operate on the data as a sequence of bytes, and grouping corresponding bytes facilitates the recognition of repeating patterns in the data flow.

At high precisions the compression algorithms do not take advantage of the correlated signal and both signals have the same compression ratio, except for lzma, which is the most expensive of all three. The difference between the signals is also small for 16-bit linear packing. At lower precisions the correlated array compresses notably better than the uncorrelated one.

Compression of linearly packed data does only marginally better for the correlated array at 16 bits, while it makes a notable difference between the arrays at 8-bit resolution. Linear packing of the uncorrelated array at 16 bits leads to some $15 \%$ better $z$ lib compression than corresponding precision trimming; for the correlated array the difference is much smaller. In all other cases the precision-trimming results in the same or better compressions than the corresponding shuffled packed array.

\section{Practical examples}

Lossy compression is a compromise between the accuracy and the compression efficiency. Optimal parameters of compression depend on the nature of the data and on the intended application. In this section we give examples on suitable compression parameters for several practical applications. For illustrations we use some atmospheric variables that the author dealt with.

Consider gridded fields of the $2 \mathrm{~m}$ temperature and the surface-air pressure in the Earth atmosphere. The fields have a limited dynamic range: the values of pressure fall within $330 \mathrm{hPa}$ (at the Everest summit) and $1065 \mathrm{hPa}$ (at Dead Sea level), and for the temperature the range is 200 to $360 \mathrm{~K}$. For such fields the spacing of quantization levels for precision trimming falls within a factor of $2-4$, and to achieve the same norm of absolute (or relative) precision one would need approximately the same number of mantissa bits for precision trimming or bits per value for linear packing. The fields are usually smooth, so the most significant bits do not differ among neighboring cells, and a subsequent lossless compression yields a substantial gain in size even with a large number of bits kept. 
(a) zlib, linear scale

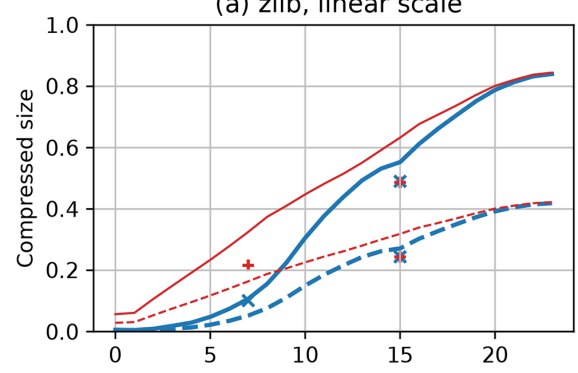

(c) bzip2, linear scale

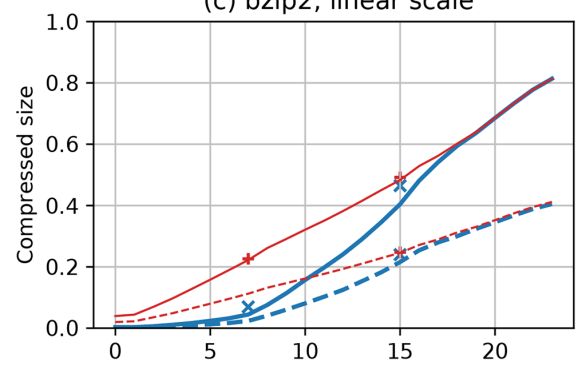

(e) Izma, linear scale

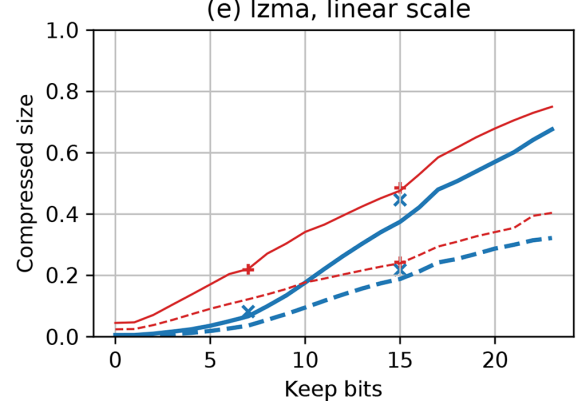

(b) zlib, log scale

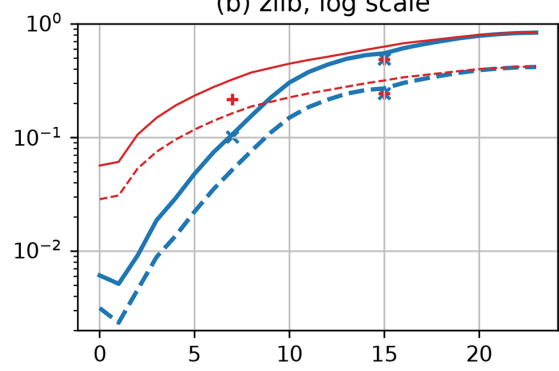

(d) bzip2, log scale

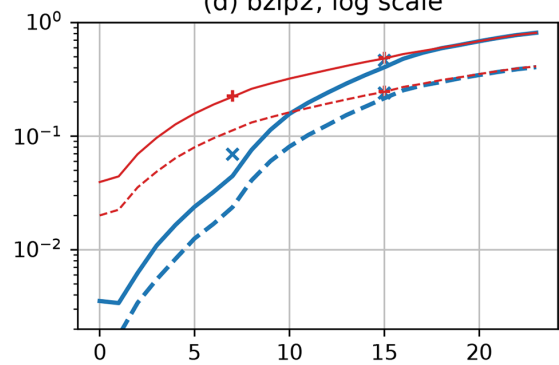

(f) Izma, log scale

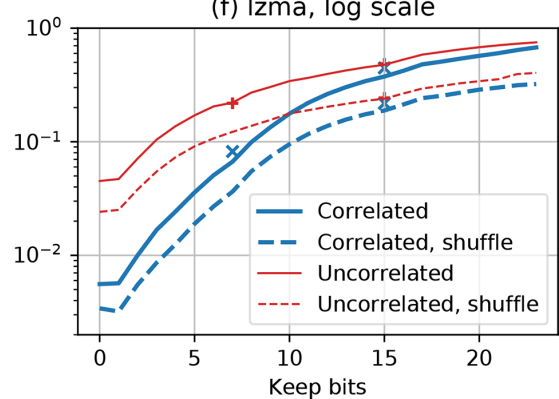

Figure 5. The normalized compressed size (compression ratios) of the arrays shown in Fig. 2, as a function of the number of keep bits for mantissa rounding with and without shuffling. Compression ratios for same arrays packed with linear packing are shown with points of respective color. The ratios are shown both in linear and logarithmic scale for three compression methods. Twenty-three keep bits correspond to the original precision.

Suppose the intended use of the dataset is the calculation of the components of a geostrophic wind $u_{g}, v_{g}$, defined as (Holton and Hakim, 2013)

$-f v_{g} \simeq-\frac{1}{\rho} \frac{\partial p}{\partial x} ; \quad-f u_{g} \simeq-\frac{1}{\rho} \frac{\partial p}{\partial y}$,

where $f \simeq 10^{-4} \mathrm{~s}$ is a Coriolis parameter and $\rho$ is the air density. If the target accuracy for the geostrophic wind is $0.1 \mathrm{~m} \mathrm{~s}^{-1}$ or $1 \%$ and the grid spacing is $10 \mathrm{~km}$, one would need the pressure accurate within $0.01 \mathrm{hPa}$ and temperature within $2 \mathrm{~K}$. Therefore 17 mantissa bits will be needed for the pressure, while only 6 bits are needed for the temperature. However, if both fields are needed to convert between the volume mixing ratio and the mass concentration of an atmospheric trace gas, whose measurements are accurate within, e.g., $1 \%$, six to seven bits per value will be sufficient for both variables.

Another example is wind components from a meteorological model. For practical applications, like civil engineering or wind energy one would need a precision exceeding the precision of reference wind measurements. Best atmospheric cup anemometers have about $1 \%$ relative accuracy and about $1 \mathrm{~cm} \mathrm{~s}^{-1}$ absolute accuracy. If we neglect representativeness error, which is usually larger than an instrument error but less trivial to evaluate, one would need six to seven mantissa bits for the model wind components. If one is going to reconstruct the vertical wind component from the horizontal ones using the continuity equation, e.g., for offline dispersion modeling, the required precision of horizontal components is absolute and depends on the grid resolution, similarly to the aforementioned case of geostrophic wind.

One could note that relative-precision trimming leaves excess precision for small absolute values of wind and is therefore suboptimal for data compression. The absoluteprecision trimming should be more appropriate for wind components. Since the range of wind components in the atmosphere is limited, linear packing has been successfully used for wind components without major issues. Archives 
of numerical weather prediction (NWP) forecasts typically use linear packing with 16-18 bits per value for wind components.

In the aforementioned case of a concentration field originating from a point source, where LP is inapplicable, precision trimming works well. Precision trimming does not affect the representable dynamic range of the field, and the relative precision of the integral of the field over some areas is at least the same as one of the precision-trimmed data.

Often, in problems of atmospheric dispersion, both the emission sources and observations have quite large uncertainty; therefore there is no reason to keep a large number of significant figures in simulation results. If one creates a lookup database for scenarios of an emergency release of hazardous materials at various meteorological conditions, the database size is one of the major concerns, while the uncertainty of the released amount is huge. For such applications even 1-2 keep bits for the concentration fields can be sufficient.

For model-measurement comparisons of such simulations one might be interested only in concentrations exceeding some threshold, e.g., some fraction of the detection limit of the best imaginable observation. Then in addition to trimming relative precision, one could trim the absolute precision of the field and therefore zero-out a substantial part of the model domain, further reducing the dataset storage size.

\section{Conclusions}

A simple method for trimming precision by rounding a mantissa of floating-point numbers has been implemented and tested. It has been incorporated into the NCO mainstream and has been used by default since $\mathrm{v}$ 4.9.4. The method has half the quantization error of the Bit Grooming method (Zender, 2016), which was used by default in earlier versions of NCO. Bit Grooming, besides having suboptimal precision, leads to substantial distortion of multipoint statistics in scientific datasets. The "halfshave" procedure can be used to partially recover the precision and remove excess distortions from two-point statistics of bit-groomed datasets.

Precision trimming should be applied to data arrays before feeding them to NetCDF or any another data-output library. The trimming can be applied to any data format, e.g., raw binary, that stores arrays of floating-point numbers to facilitate subsequent compression. NCO provides a limited leverage to control the number of mantissa bits in terms of specifying "a number of significant digits" (SD) for a variable. These digits can be loosely translated into the number of mantissa bits: $1 \mathrm{SD}$ is 6 bits, $2 \mathrm{SD}$ is 9 bits, $3 \mathrm{SD}$ is 12 bits, etc. The exact translation varies slightly among the NCO versions; therefore low-level data processing should be used if one needs to control the exact number of mantissa bits to keep.

Along with a relative-precision trimming that keeps a given number of mantissa bits and therefore guarantees a given relative precision, we considered absolute-precision trimming that specifies a value for the least significant mantissa bit. The latter method is recommended when required absolute precision is specified. Depending on the nature of a variable in the dataset and intended application either or both of the trimmings can be applied to remove non-significant information and achieve the best compression performance.

Precision trimming and subsequent lossless compression has substantial advantages over the widely used linear packing method: it allows us to explicitly specify the required precision in terms of both absolute and relative precision, guarantees to keep the sign and a valid range of an initial value, and allows for the use of the full range of floating-point values. Our examples illustrate that linear packing can lead to unconstrained errors and does not provide substantial savings in the storage space over precision trimming; therefore linear packing should be considered deprecated. The exception is GRIB2, where linear packing is applied to individual 2D fields of known dynamic range, uses unsigned integers of arbitrary length, and involves specialized compression algorithms.

The precision-trimming methods described in the paper were implemented in Python, and corresponding subroutines are given in the Appendix, where we also put subroutines for relative- and absolute-precision trimming with mantissa rounding, implemented with Fortran 95 intrinsics. The subroutines should be directly usable in various geoscientific models. 


\section{Appendix A: Implementation of precision trimming}

In the Appendix we list simple subroutines that implement shave, halfshave, and two versions of round precision trimming. As is pointed out in the Conclusions, rounding half to even should be used for precision trimming unless there is a good reason to apply another method. The subroutines are implemented in Python/numpy and can be embedded directly in data-handling software. Also, rounding implemented in Fortran 95 without explicit bitwise operations is given below.

All subroutines receive an array a of single-precision floats to process and an integer keepbits specifying the required number of keep bits. The subroutines can be adapted to handle double-precision floats as well.

We assume that all the parameters are within a valid range: the number of keep bits is within the range from 1 to 24 , and the array values are finite and not equal to any value with a special meaning in the context of the application (e.g., missing value).

\section{A1 Bit shave}

Here a simple mask zeroing tail bits is applied to the array.

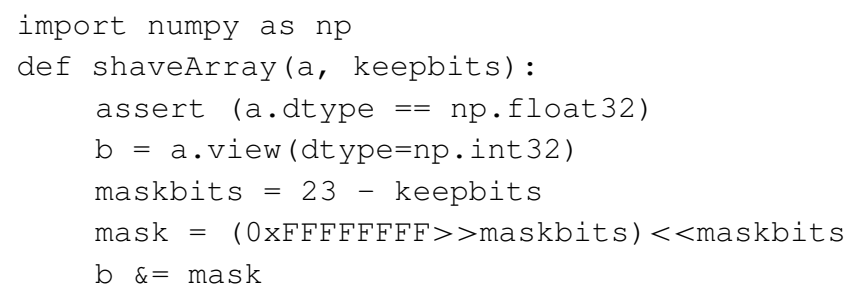

\section{A2 Halfshave}

This is the same as above, but the most significant of tail bits is set.

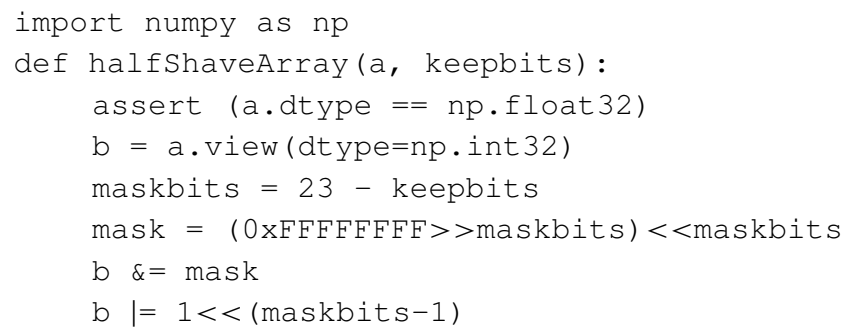

\section{A3 Rounding, half to infinity}

Here we use an integer operation to add a half-quantum before bit shaving.

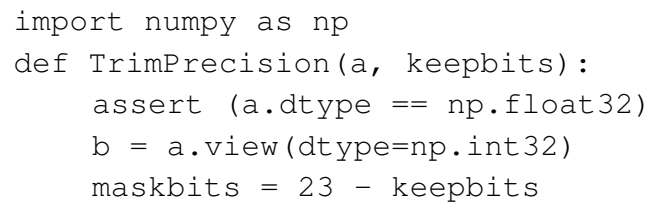

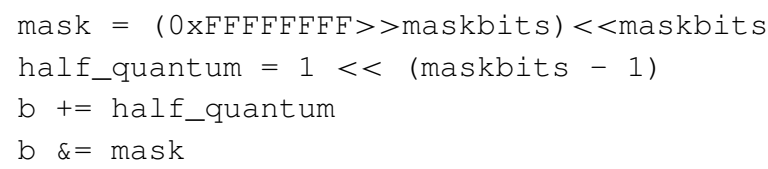

\section{A4 Rounding, half to even}

To implement half to even, before bit shaving we add a halfquantum to those values that have 1 in the least significant of keep bits, and they are rounded half to infinity as in the previous example. If the least significant of keep bits is 0 , we add less than a half-quantum, so bit-shaving results in rounding half to 0 . This code was inspired by Milan Klöwer (personal communication, July 2020).

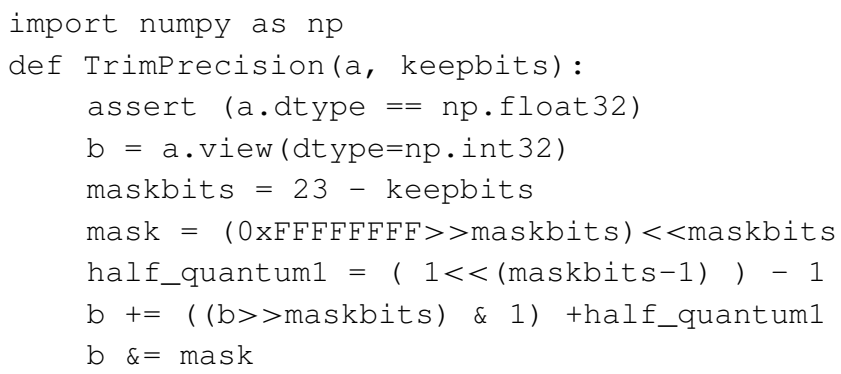

\section{A5 Rounding, half to even in Fortran 95}

Since type casting is not reliable in some versions of Fortran, here is an implementation that uses only standard Fortran 95 intrinsics. It is probably not as efficient computationally as an implementation via bitwise and integer operations, but the cost of this operation should be negligible in a context of any geophysical model. The subroutine uses the default rounding mode of Fortran, which is half to even, according to IEEE 754.

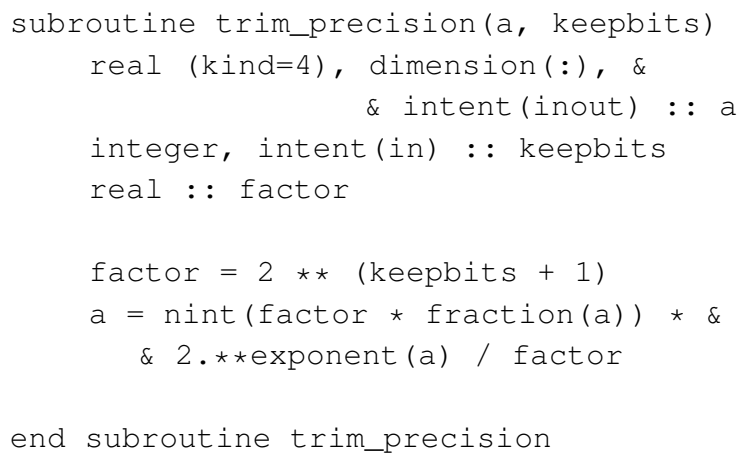

\section{A6 Rounding to given absolute precision in Fortran 95}

In this example max_abs_err specifies the limit for absolute error for each individual value. The resulting absolute error is within a half of quantum, which is set to the integer power of 2 to ensure that tail bits are set to 0 . As in the previous example the half-to-even IEEE 754 rounding produces the unbiased result. 


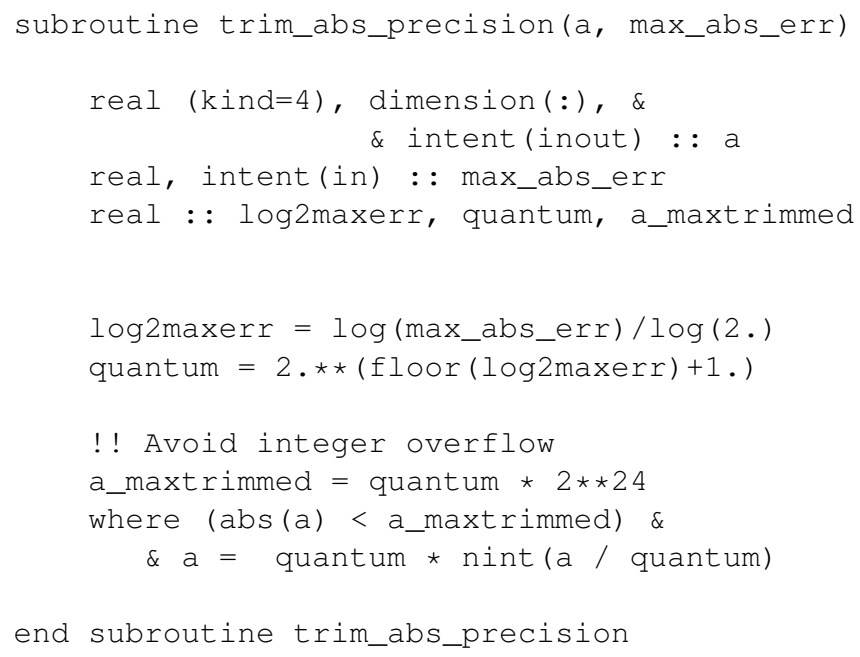


Code availability. The implementation of mantissa rounding in $\mathrm{C}$ has been introduced into the NCO master branch. The source code of NCO is available from http://nco.sourceforge.net (last access: 7 December 2020) under BSD license. The Python3 code used to generate the figures and the example statistics is available from the Supplement and can be used under the BSD license.

Supplement. The supplement related to this article is available online at: https://doi.org/10.5194/gmd-14-377-2021-supplement.

Competing interests. The author declares that there is no conflict of interest.

Acknowledgements. I would like to thank Mikhail Sofiev and Viktoria Sofieva from the Finnish Meteorological Institute and Dmitrii Kouznetsov from the University of ElectroCommunications, Tokyo, for fruitful discussions and Charles Zender from the University of California for the NetCDF Operator software (NCO) and for being open and swift about verifying and accepting the patches for it. Also, I am grateful to Milan Klöwer from Oxford University, Mario Acosta from the Barcelona Supercomputer Center, Seth McGinnis from the National Center for Atmospheric Research, and Ananda Kumar Das from the Indian Meteorological Department for their careful reading of the discussion paper and their critical and valuable comments. The initial implementation of mantissa rounding for the SILAM model was done within the "Global health risks related to atmospheric composition and weather, GLORIA" project from the Academy of Finland (grant 310373). Adaptation of the mantissa rounding for absolute precision was made for the Russian Foundation for Basic Research "Experimental studies of internal gravity waves in the mid-altitude atmospheric boundary layer" (project no. 1905-01008). The detailed analysis of the precision trimming was performed within the service contract CAMS_61 "Development of regional air quality modelling and data assimilation aspects" of the Copernicus Atmosphere Monitoring Service (contract notice 2019/S 118-289635).

Review statement. This paper was edited by Paul Ullrich and reviewed by Ananda Kumar Das, Mario Acosta, and Seth McGinnis.

\section{References}

ANSI/IEEE: IEEE Standard for Binary Floating-Point Arithmetic, ANSI/IEEE Std 754-1985, pp. 1-20, https://doi.org/10.1109/IEEESTD.1985.82928, 1985.

Holton, J. and Hakim, G.: An Introduction to Dynamic Meteorology, Academic Press, Elsevier Science, Amsterdam, Boston, Heidelberg, London, New York, Oxford, Paris, San Diego, San Francisco, Singapore, Sydney, Tokyo, 2013.

Stackpole, J. D.: GRIB (Edition 1) The WMO Format for the Storage of Weather Product Information and the Exchange of Weather Product Messages in Gridded Binary Form, Office note 388, U.S. Department of Commerce, National Oceanic and Atmospheric Administration, National Weather Service, National Meteorological Center, MD, USA, 1994.

Zender, C. S.: Bit Grooming: statistically accurate precisionpreserving quantization with compression, evaluated in the netCDF Operators (NCO, v4.4.8+), Geosci. Model Dev., 9, 3199-3211, https://doi.org/10.5194/gmd-9-3199-2016, 2016. 